\title{
Modal characteristics of two operating power transmission poles
}

\author{
Shen-En Chen* and Kaoshan Dai \\ Department of Civil and Environmental Engineering, University of North Carolina at Charlotte, 9201 University \\ City Boulevard, Charlotte, NC 28223 - 0001, USA
}

\begin{abstract}
Unique conductor-pole couplings complicate the dynamic behaviors of electric transmission pole line systems. Finite element modeling is performed on two typical transmission poles used in southeastern USA - a steel pole and a prestressed concrete pole. The two poles are representative of unique structure types: a heavy rod-like structure and a lightweight, shell-type structure. Since coupling issues between the pole and the cable introduce great complexities for modeling the pole line system, simplified numerical models are used. Limited full-scale modal test results are presented to verify the numerical models. The prestressed concrete pole is shown to be easier for mode identification than the steel pole - but both numerical models show complicated coupled vibration modes. This study is part of a larger study to establish an understanding of the dynamic response analyses of power grid under ground vibrations.
\end{abstract}

Keywords: Finite element modeling, prestressed concrete poles, vibration characteristics

\section{Introduction}

Electric transmission lines are unique civil infrastructure for power delivery that typically supported by either transmission towers or pole-type structures. In southeastern USA, transmission poles are used extensively in the electric grids. Due to reduced redundancy, these pole structures are vulnerable to extreme event loadings, such as strong ground motions due to earthquake or blasting. Structural protection strategies for strong ground motions rely mostly on engineering experiences to establish ground vibration limits, which are based on previous studies done on residential structures [1]. Such approach ignored actual dynamic responses of the structures, and is not consistent with other strong ground motion studies, i.e. earthquakes, where structural resonant vibration modes must be determined. There are currently no specific investigations or reports about strong ground motion effects on pole type structures. The work reported in this paper describes the study of dynamic behaviors of two operating transmission poles using Finite Element (FE) analysis.

Most vibration studies on transmission line systems are conducted on transmission towers under wind loads [2, 3]. El-Attar [4] evaluated current design codes for transmission lines under earthquakes and modeled both power lines and steel towers. In the study, seismic responses were calculated and compared with the effects of wind and ice loads. This study showed that displacements and internal forces in transmission lines are substantial under ground vibrations, and the dynamic effects may exceed those caused by the wind loads. Ghobarah et al. [5] studied transmission towers subjected to spatially incoherent seismic ground motions - they found that seismic wave velocities had significant effects on lateral displacements of transmission lines. The complexity caused by the coupling between cables and supporting structures increases the difficulty in studying dynamic responses of transmission systems. Loredo-Souza [6] examined the behavior of transmission lines under severe winds through wind tunnel tests and found that aerodynamic damping had significant effects on dynamic behaviors of cables. He

\footnotetext{
*Corresponding author. E-mail: schen12@uncc.edu.
} 
concluded that with increasing separations between cables, coherence between cable forces diminished. McClure and Lapointe [7] summarized their work on cable dynamic analysis under shock loads due to conductor breakages. Venkatasubramanian [8] studied cable behaviors for galloping and pointed out that galloping may be initiated in a purely vertical mode, purely torsional mode or a combination of both.

It is challenging to simulate coupled conductor/tower behaviors using full solid FE models: Cable vibration alone is difficult to model using finite element method [9]. Hence, simplified models, which take into account the coupling characteristics, have been developed for transmission tower-line systems [10,11]. Li et al. [12] proposed a simplified method to compute seismic responses of coupled tower-conductor systems by adding attached discrete conductor masses.

Studies of the free vibration of transmission pole structures are limited: Lantrip [13] conducted modal tests on several concrete pole structures under free-free boundary conditions and developed distributed mass models using ANSYS ${ }^{\circledR}$; Chen et al. [14] identified vibration modes of prestressed concrete poles using both modal testing and FE simulation and for both free-free and cantileveled conditions; Polyzois [15] used FE method to obtain natural frequency of composite poles; finally, Dai and Chen [16] reported the influence of prestress effects on modal behaviors of prestressed concrete poles.

This paper presents both numerical and experimental studies on the modal behaviors of two transmission poles under operation: one is a prestressed concrete pole and the other is a steel pole. The discussion focuses on the numerical simulation of modal behaviors, including the simplified model proposed by Li et al. [12], which is explored to reduce the full-scale model of the two poles in order to accommodate the conductor/structure coupling effects. Free vibrations of the coupled transmission pole-line systems calculated with different models are also compared.

\section{Modeling of coupled systems}

Field experimentations to study the interactions between support structure and cables in a transmission grid are rarely conducted. This is because the unique long-span feature of the transmission lines limits the extent of physical experiments. The cost and safety in field tests also make it prohibitive for large-scale experimental works. Therefore, numerical analysis has been widely used by researchers to study dynamic characteristics of the transmission structure.

However, detailed numerical modeling can be expensive and time consuming; as a result, Li suggests to include the coupling effects for out-of-plane (perpendicular to the conductor cable) motions by modifying the system rigidity matrix, and for in-plane (parallel with the conductor cable) motions by modifying the mass matrix [12]:

out-of-plane vibration:

$$
\begin{aligned}
{[M] } & =\left[[M]_{\text {line }}[M]_{\text {tower }}\right]^{T} \\
{[K] } & =\left[\begin{array}{ll}
{[K]_{\text {line }}} & {[K]_{\text {coupling }}} \\
{[K]_{\text {coupling }}^{T}[K]_{\text {tower }}}
\end{array}\right]
\end{aligned}
$$

in-plane vibration:

$$
\begin{aligned}
{[M] } & =\left[\begin{array}{ll}
{[M]_{\text {line }}} & {[M]_{\text {coupling }}} \\
{[M]_{\text {coupling }}^{T}} & {[M]_{\text {tower }}}
\end{array}\right] \\
{[K] } & =\left[\begin{array}{ll}
{[K]_{\text {line }}[0]} \\
{[0]^{T}} & {[K]_{\text {tower }}}
\end{array}\right]
\end{aligned}
$$

where $[M]$ and $[K]$ are the mass and stiffness matrices of the coupled system; $[M]_{\text {tower }}$ and $[K]_{\text {tower }}$ are the mass and stiffness matrices of the tower; $[M]_{\text {line }}$ and $[K]_{\text {line }}$ are the mass and stiffness matrices of the cables; and $[M]_{\text {coupling }}$ and $[K]_{\text {coupling }}$ are the mass and stiffness matrices generated by tower-line interactions, respectively. 


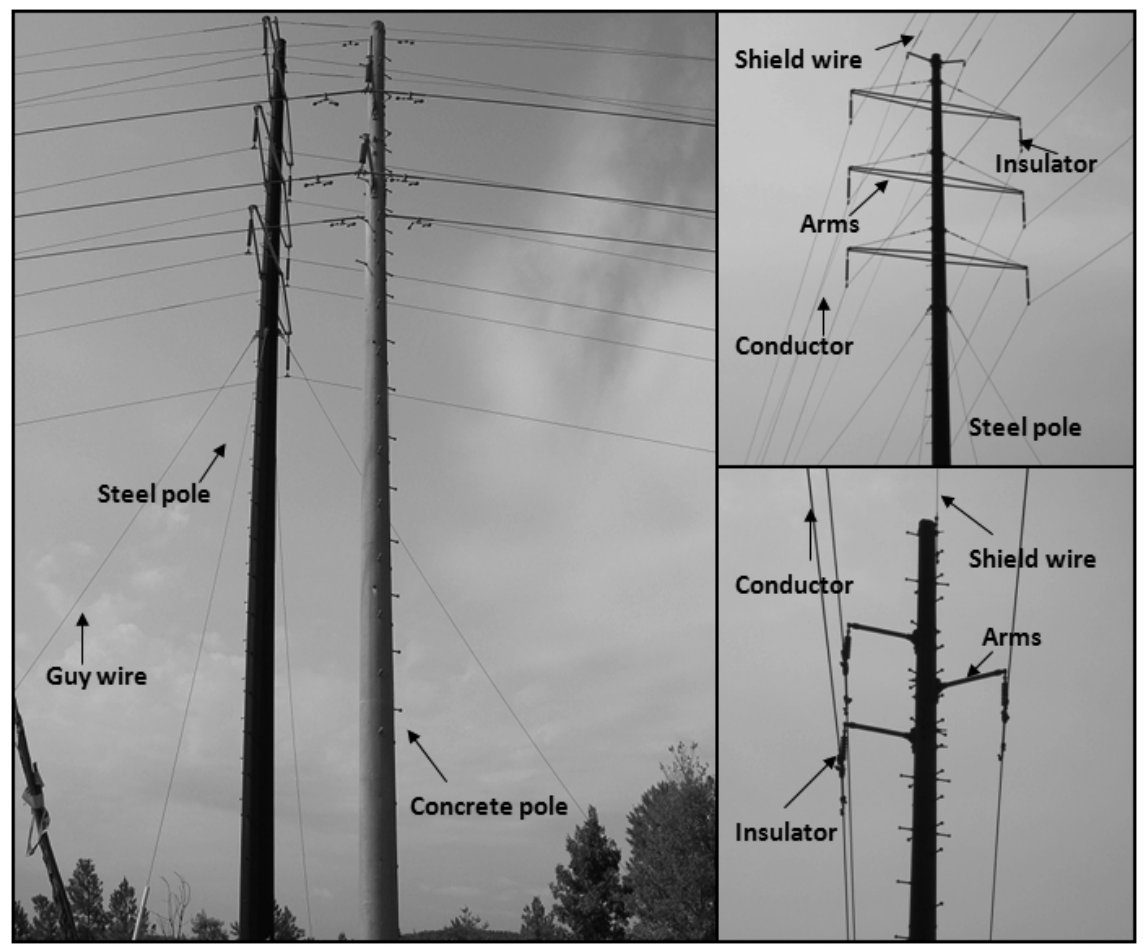

Fig. 1. Two operational transmission lines studieds.

\section{Experimental study of coupled transmission pole-line system}

In this paper, two in-operation pole-supported transmission lines are studied (Fig. 1). The steel pole is part of a $115 \mathrm{kV}$ double-circuit transmission line. The concrete pole is the supporting structure in an operating $46 \mathrm{kV}$ line. These two lines are in parallel and in close distance to each other. The steel pole is supported with four guy cables. The total height of the concrete pole is $18.29 \mathrm{~m}$; while the steel pole is $32.01 \mathrm{~m}$. Both poles are directly embedded with a depth of 10 percent of the pole length plus $0.61 \mathrm{~m}$.

Impact modal tests were conducted on both pole structures with one low-frequency piezoelectric accelerometer (PCB model 393B12) attached to the pole, multiple-point impacts were exerted along the pole with a $53 \mathrm{~N}$ instrumented hammer (ICP impulse force test hammer). During the impact tests, the lines were temporarily powered off and the hammer impacts were administered via a mechanical lift. The free vibration behaviors of the poles are then extracted from the Frequency Response Functions (FRFs). The identified modes and their associated natural frequencies are listed in Table 1. The levels of difficulties to extract modal frequencies and FRFs are different between the two structures: The prestressed concrete pole behaved as a heavy rod-type structure with constrained boundaries, where clear vibration modes can be identified. The steel pole behaves as a thin-shell structure with strong coupling with the conductors and the guy wires. As a result, its modal behaviors are not very well observed. In fact, only one mode was identified in our experimental study.

To obtain the soil properties, geophysical testing using shear wave velocity measurements [17] was performed at the site where the two poles were embedded. NEHRP site classification [18] was referred to define soil property of the testing location. The resultant $\overline{V_{s}}$ is $451.27 \mathrm{~m} / \mathrm{s}$, which indicates very dense soil at the testing site.

\section{Numerical analysis of the coupled transmission pole-line systems}

To simulate the modal behaviors, FE normal mode (eigen-value) analysis of the two operational transmission poles was conducted. Table 2 lists the different models constructed with varied levels of details. 
Table 1

Eigenvalue comparison between different models

\begin{tabular}{|c|c|c|c|c|c|c|c|c|}
\hline \multirow[t]{2}{*}{ Testing results or models } & \multicolumn{2}{|c|}{$1^{\text {st }}$ bending } & \multicolumn{2}{|c|}{$2^{\text {nd }}$ bending } & \multicolumn{2}{|c|}{$3^{\text {rd }}$ bending } & \multicolumn{2}{|c|}{$4^{\text {th }}$ bending } \\
\hline & $\mathrm{In}^{*}$ & Out* & $\mathrm{In}^{*}$ & Out* & $\operatorname{In} *$ & Out* & $\operatorname{In}^{*}$ & Out* \\
\hline Concrete pole modal testing & - & - & 6.10 & 6.71 & 15.26 & 17.09 & 30.52 & 31.74 \\
\hline CPA & 1.49 & 1.49 & 6.79 & 6.79 & 17.18 & 17.18 & 32.46 & 32.46 \\
\hline $\mathrm{CPB}$ & - & - & 6.06 & 6.65 & 15.64 & 16.68 & 31.99 & 32.07 \\
\hline $\mathrm{CPC}$ & 1.17 & 1.32 & 6.15 & 6.45 & 14.87 & 16.03 & 27.78 & 29.75 \\
\hline Steel pole modal testing & - & - & - & - & 8.54 & - & - & - \\
\hline SPA & 1.20 & 1.20 & 4.92 & 4.92 & 11.99 & 11.99 & 22.36 & 22.36 \\
\hline SPB & - & - & 7.41 & 7.41 & 12.01 & 12.01 & 22.83 & 22.83 \\
\hline SPC & - & - & 6.54 & 6.55 & 9.27 & 10.51 & 20.94 & 23.82 \\
\hline SPD & 0.60 & 0.78 & 3.37 & 3.97 & 8.26 & 9.38 & 15.51 & 18.20 \\
\hline SPE & - & - & 5.13 & 6.23 & 8.99 & 9.64 & 15.70 & 18.64 \\
\hline
\end{tabular}

* In - in-plane vibration; Out - out-of-plane vibration; unit: Hz.

Table 2

FE models used in numerical analysis

\begin{tabular}{ll}
\hline Model & Structural configuration \\
\hline CPA & FE model of the isolated concrete pole \\
CPB & FE model of the concrete pole-line system \\
CPC & FE model of the isolated concrete pole with added conductor mass \\
SPA & FE model of the isolated steel pole \\
SPB & FE model of the guyed steel pole \\
SPC & FE model of the steel pole-line system \\
SPD & FE model of the isolated steel pole with added conductor mass \\
SPE & FE model of the guyed steel pole with added conductor mass \\
\hline
\end{tabular}

The coupled transmission line sections with two separate spans of conductors in the models were created either through detailed modeling (poles, all cables, insulators, and arms, all included), or without cables in the model, or using the simplified model proposed by Li et al. [12] with added masses:

$$
\Delta m=f(l) \times l \times q
$$

where $\Delta m$ is the additional mass of the pole considering the conductor effects; $l$ is the horizontal distance between two poles; $q$ denotes the conductor mass per unit length; and $f(l)$ is a factor determined from the following equations [12]:

for out-of-plane

$$
f(l)=\left\{\begin{array}{ll}
0.17+3 l / 200 l_{0} & \text { soft } \\
0.21+l / 100 l_{0} & \text { mid-hard } \\
0.35+l / 20 l_{0} & \text { hard }
\end{array} \quad \text { if } f(l)>0.7, \text { then } f(l)=0.7\right)
$$

for in-plane

$$
\left.f(l)=0.5+\frac{l}{200 l_{0}} \text { at all sites (if } f(l)>1.0, \text { then } f(l)=1.0\right)
$$

where $l_{0}$ is the limit span. When the span exceeds this value, cable effects need to be considered. For mid-point site, $l_{0}=200.00 \mathrm{~m}$.

ANSYS ${ }^{\circledR}$ was used for constructing the FE models [19]. The pole structures were modeled with tapered beam elements (BEAM189). For the coupled pole-line systems: the cables, including conductors and shield wires, were modeled with tension-only truss elements (LINK10); insulators were generated with uniaxial spar elements (LINK8); and the arms that connect the insulators and the concrete pole were modeled with elastic beam elements (BEAM4). Link elements (LINK8) were used to model the steel pole arms with consideration of its joint connection characteristics. Mass elements (MASS21) were added at the nodes where the cables are connected to the main structure in the simplified model (CPC). Table 3 listed the properties of the two poles used in the modeling. In CPC, SPD, and SPE models, additional mass factors are calculated from the mid-hard soil scenario using Eqs (4) and (5). 


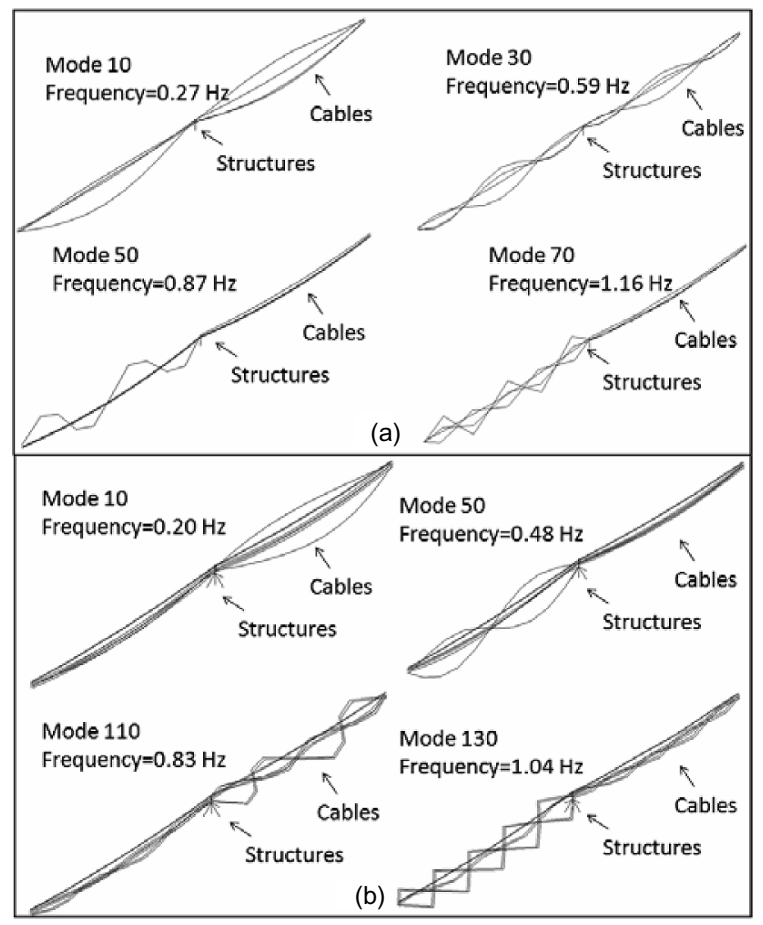

Fig. 2. Representative cable-leading modes of the coupled system: (a) concrete pole; (b) steel pole.

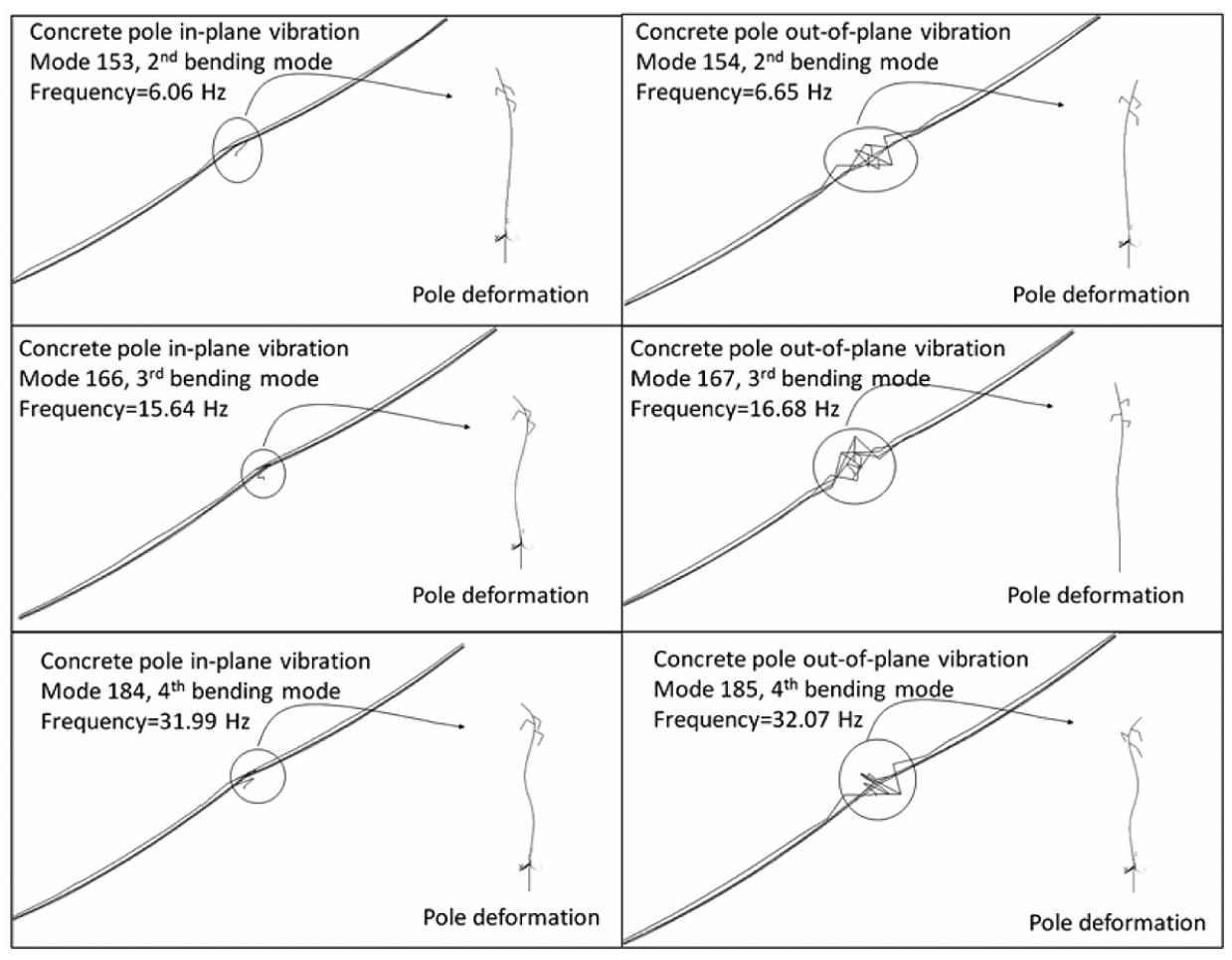

Fig. 3. Representative pole-leading modes of the coupled concrete pole-line system. 
Table 3

Parameters of the concrete and steel pole-line systems

\begin{tabular}{|c|c|c|c|}
\hline Concrete pole parameters & & Steel pole parameters & \\
\hline Overall length (m) & 18.29 & Overall length $(\mathrm{m})$ & 32.00 \\
\hline Embedment depth (m) & 2.44 & Embedment depth (m) & 3.81 \\
\hline Density $\left(\mathrm{kg} / \mathrm{m}^{3}\right)$ & 2564.57 & Density $\left(\mathrm{kg} / \mathrm{m}^{3}\right)$ & 7853.98 \\
\hline Modulus of elasticity (GPa) & 41.52 & Modulus of elasticity (GPa) & 199.95 \\
\hline Line parameters & & Line parameters & \\
\hline Left span length (m) & 322.78 & Left span length (m) & 333.15 \\
\hline Difference in elevation at the left span (m) & 6.71 & Difference in elevation at the left span (m) & 7.05 \\
\hline Right span length (m) & 341.07 & Right span length (m) & 347.17 \\
\hline Difference in elevation at the right span (m) & & Difference in elevation at the right span (m) & 19.10 \\
\hline Conductor parameters & & Conductor parameters & \\
\hline Nominal cross-section diameter (mm) & 18.80 & Nominal cross-section diameter (mm) & 14.48 \\
\hline Weight per unit length (kg/m) & 0.64 & Weight per unit length $(\mathrm{kg} / \mathrm{m})$ & 1.15 \\
\hline Modulus of elasticity (GPa) & 59.98 & Modulus of elasticity (GPa) & 59.98 \\
\hline Shield wire parameters & & Shield wire parameters & \\
\hline Nominal cross-section diameter (mm) & 9.65 & Nominal cross-section diameter (mm) & 11.68 \\
\hline Weight per unit length (N/m) & 0.40 & Weight per unit length $(\mathrm{kg} / \mathrm{m})$ & 0.37 \\
\hline Modulus of elasticity (GPa) & 177.88 & Modulus of elasticity (GPa) & 177.88 \\
\hline Insulator parameters & & Guy wire parameters & \\
\hline Overall weight $(\mathrm{N})$ & 17.79 & Nominal cross-section diameter (mm) & 12.70 \\
\hline Estimated modulus of elasticity (GPa) & 44.99 & Weight per unit length $(\mathrm{kg} / \mathrm{m})$ & 0.98 \\
\hline Arm parameters & & Modulus of elasticity (GPa) & 177.10 \\
\hline Overall weight per arm combination connection $(\mathrm{N})$ & 5.34 & Insulator parameters & \\
\hline \multirow[t]{5}{*}{ Modulus of elasticity (GPa) } & 44.99 & Overall weight $(\mathrm{N})$ & 44.48 \\
\hline & & Estimated modulus of elasticity (GPa) & 44.99 \\
\hline & & Arm parameters & \\
\hline & & Overall weight per arm combination connection $(\mathrm{N})$ & 289.12 \\
\hline & & Modulus of elasticity (GPa) & 44.99 \\
\hline
\end{tabular}

Normal mode analysis was conducted directly for the pole-alone models (CPA, SPA) and the simplified pole-line system models (CPC, SPD). For detailed pole-line system FE models (CPB, SPC) and the guyed steel pole models (SPB, SPE), nonlinear static analysis was first conducted to sag the cable system including conductors, shield wires, and guy wires. The Block Lanczos eigenvalue solver is then performed [19]. The sag-to-span ratio was estimated from maximum sag divided by the cable span. The sag ratio values for conductors and shield wires in the CPB model are approximately 0.04 and 0.03 , respectively. In the SPC model, the sag ratio values for conductors and shield wires are 0.05 and 0.02 , respectively.

\section{Results and discussion}

\subsection{Modeling of coupled behaviors}

Numerical analysis results indicated strong coupling phenomena for both structures. Contrast to transmission tower vibration, cable vibration dominates the lower vibration modes, which is called the "cable leading modes" (shown in Fig. 2). The cable leading modes show the cable components (shield wires, conductors and guy wires) of the coupled system vibrate locally and their dynamic effects transmit to the pole structure. The main structure (pole) vibration at lower modes is not easily identified due to significant cable deformation. These "cable leading modes" play important role in the dynamic responses of the electric transmission structures under wind loads. At higher modes, however, local vibration of the pole structure dominates, which can be termed as the "pole leading modes" (Figs 3 and 4). An interesting observation is that when the vibration frequencies are close to the frequencies of uncoupled pole structure models (without cable considerations), the cables almost stay unmoved as if they are away from the main structure. The eigenfrequencies of the in-plane vibration are usually smaller than the out-of-plane counterparts of the same mode shape, which agrees well with the theory proposed by Li et al. [12]. These "pole leading modes" are of interest in the study of dynamic responses of coupled transmission structure under ground excitations. 


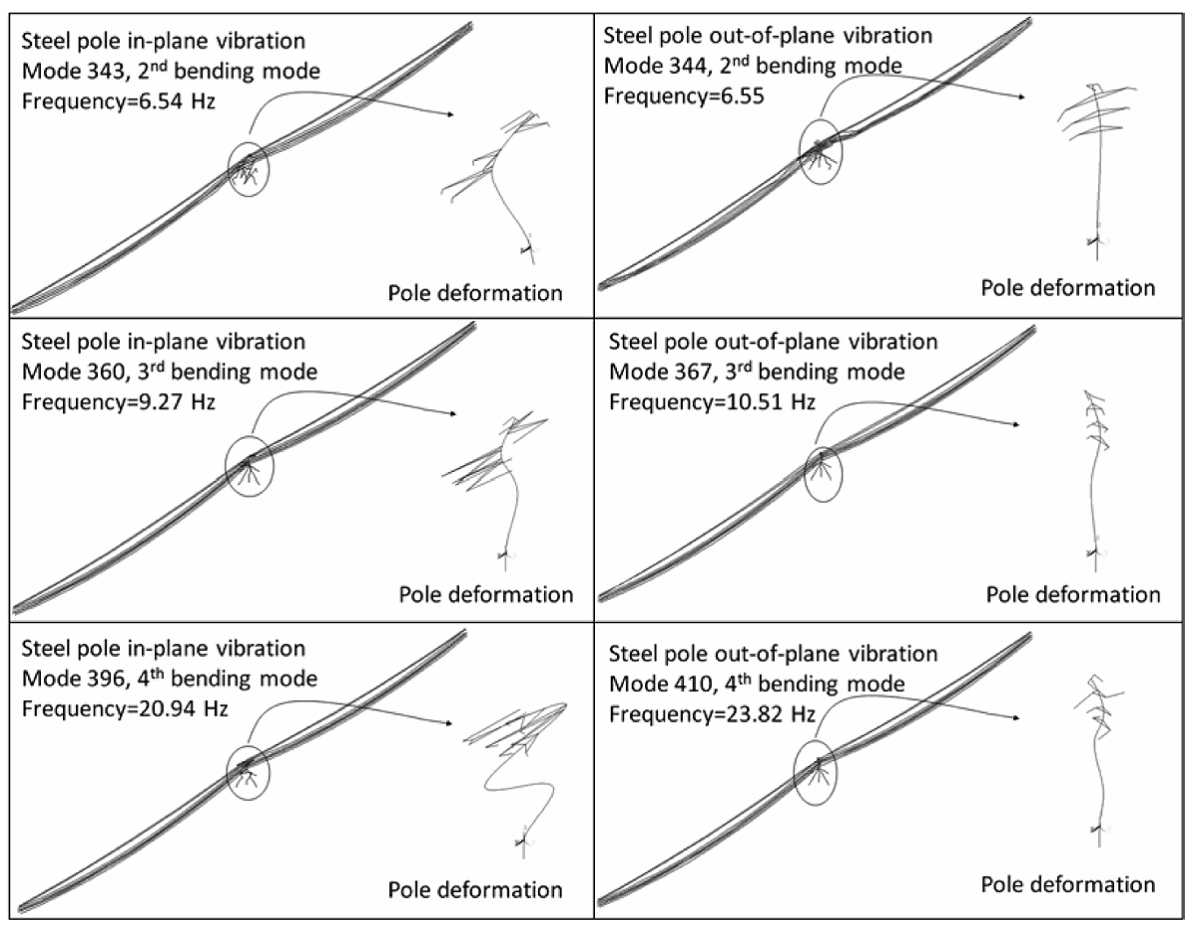

Fig. 4. Representative pole-leading modes of the coupled steel pole-line system.

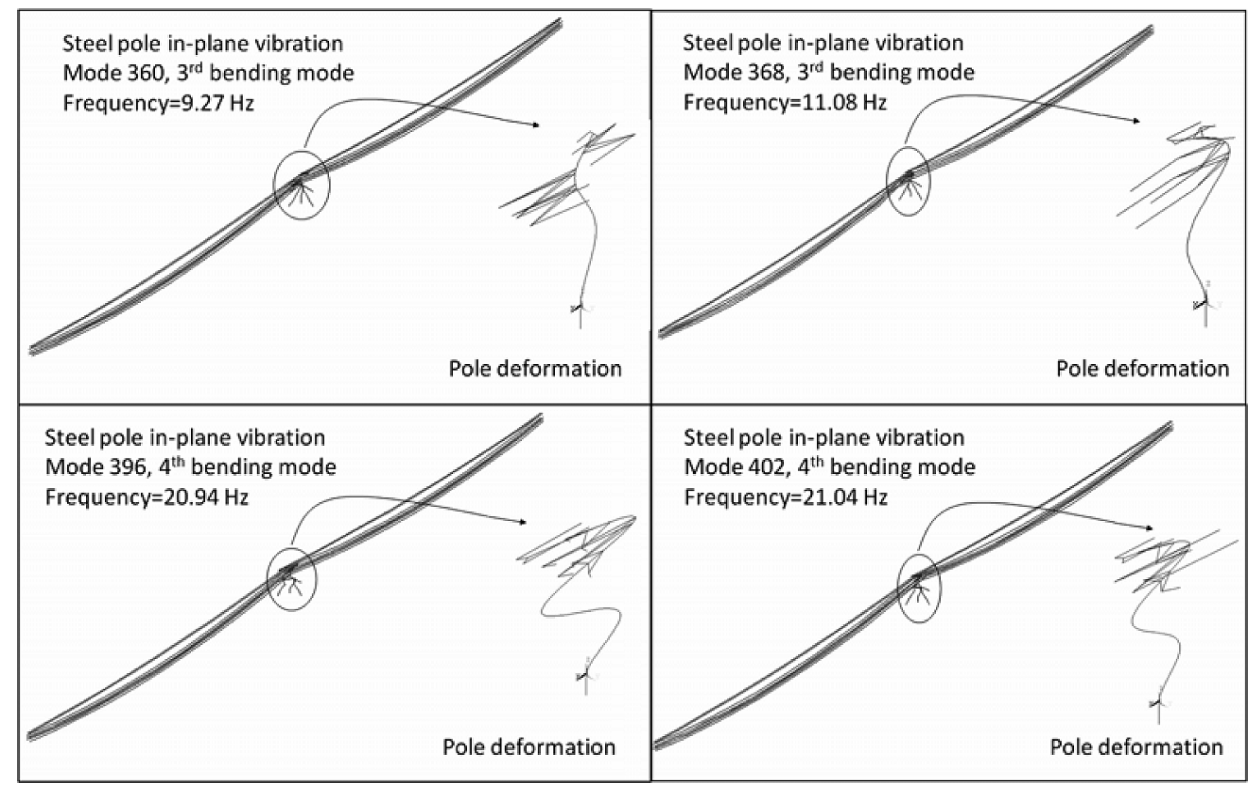

Fig. 5. Different coupled modes for steel pole structure.

The complexity of the coupling phenomena are further demonstrated by the identification of a cluster of dominant frequencies that exists around the eigensolutions of the isolated pole vibrations. There exist several possible combinations of cable-pole coupled vibration modes, which further increase the difficulty in identifying the vibration modes at lower frequencies. This phenomenon is even more complicated for the steel pole where the system involves more components (more shield wires, conductors, insulators, and with additional guy cables). Figure 5 compares 


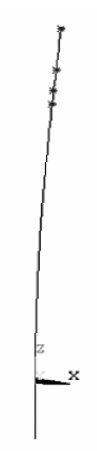

$1.17 \mathrm{~Hz}$

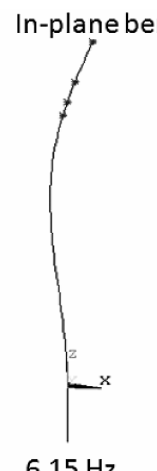

$6.15 \mathrm{~Hz} \quad 14.87 \mathrm{~Hz}$

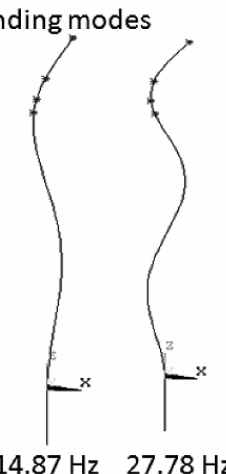

$27.78 \mathrm{~Hz}$
Out-of-plane bending modes

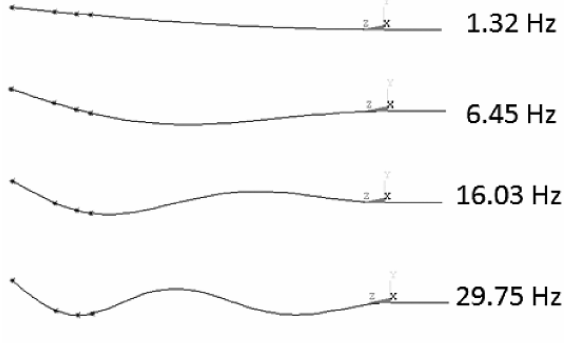

Fig. 6. First four vibration modes of the simplified concrete pole.

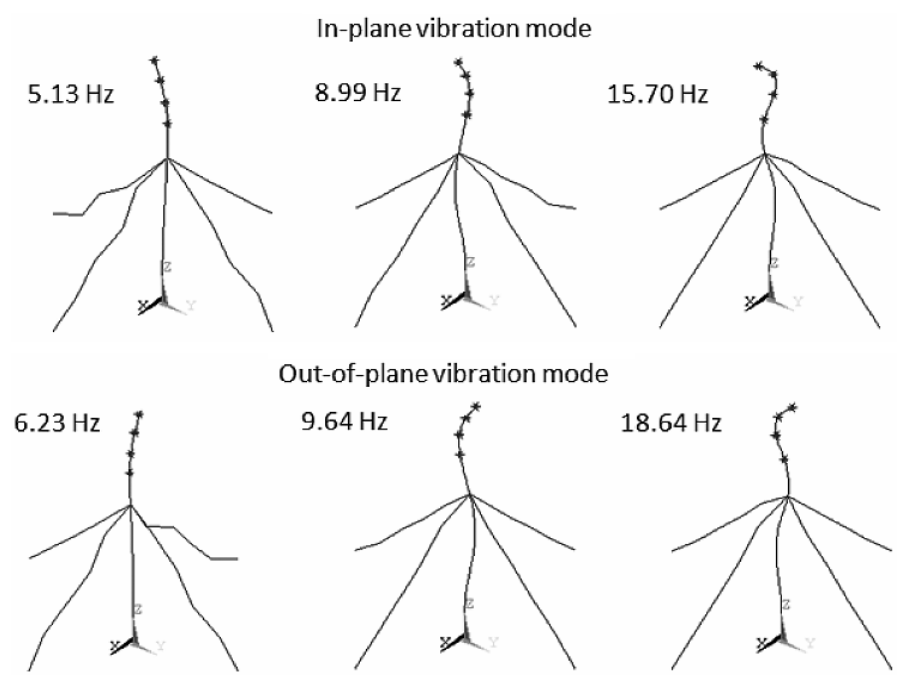

Fig. 7. Pole-leading vibration modes of the simplified steel pole.

vibration modes in which the steel pole structure has similar bending shapes while the natural frequencies varied due to different shield wire/conductor vibrations involved. Such situations generate great difficulties in designating a single frequency value for the dominant vibration mode. As a result, the frequencies for the steel pole-line system included in the figures are not unique. This coupling is also reflected in the modal experiment conducted on the steel pole, where there is little confidence identifying dominant eigen-solutions from the simple peak-picking approach. For the simplified models, as proposed by Li et al. [12], the additional masses calculated from Eqs (3-5) have been used to substitute for the shield wires and the conductors. Mass elements (MASS21) were added at the nodes where the cables connect to the main structure. Since fewer elements are required and the main structure vibration dominates in almost all modes, computation time and post analysis efforts are greatly reduced. Figure 6 shows the first four vibration modes of the simplified concrete pole model. Figure 7 gives the second to the fourth modes of the simplified steel pole model with the guy wires. Because of the coupling effect between the main structure (steel pole) and the guy cables, some lower bending modes of the steel pole have multiple frequencies, as shown in Fig. 8. Again, this coupling issue makes it difficult to claim sole eigenfrequencies for some of the vibration modes.

\subsection{Numerical and experimental results}

Table 1 summarized the eigenfrequencies obtained from both modal tests and numerical analyses. The first bending (cantilevered) modes are not detected from the modal testing of the poles. They are not included in the 


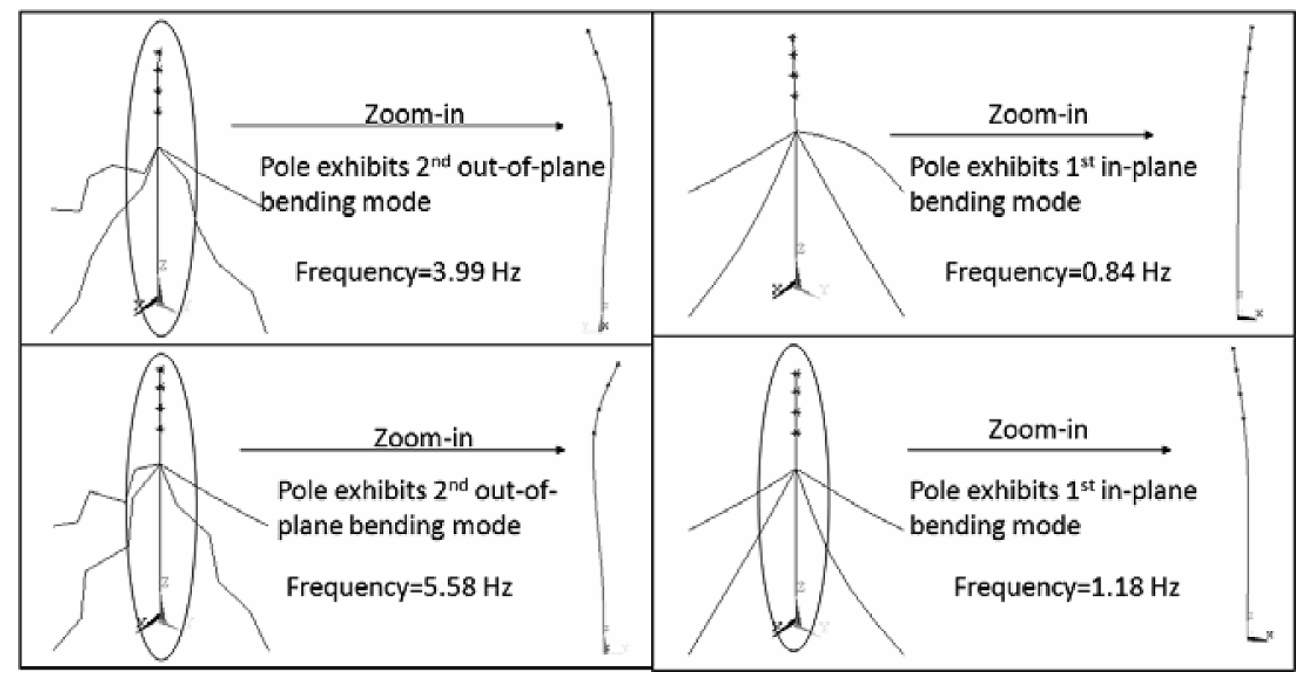

Fig. 8. Typical guy wire dominating modes for the simplified guyed steel pole.

coupled model results because the coupling effect made it difficult to identify sole frequency solutions for these modes. However, the rest of the numerical solutions for the concrete pole system correlate well with the experimental results. When the concrete pole is modeled as an isolated structure (CPA), frequency values are larger than the corresponding experimentally identified frequencies. For example, the second bending mode frequency of CPA is $11.31 \%$ different from the modal testing result. When the cable effects are included in the models (CPB), the calculated frequencies became closer to the testing results due to the addition of cable effects. This improvement indicates that, if accurate solutions are desired, only modeling the pole structure alone is not sufficient.

Simplified pole system models (CPC), built following the method proposed by Li et al. [12], show better results than the isolated pole models. The eigenfrequencies computed from the CPC model are close to both the experimental results and the solutions from the detailed coupled system model (CPB), which suggests the potential of using these models for future structural dynamic response studies.

However, the same conclusions cannot be easily drawn from comparisons between the FE models and the corresponding experimental data for the steel pole. Because of the stronger coupling phenomena, it is difficult to derive natural frequencies from the modal testing data. The eigenvalues of the steel pole as listed in Table 1 are identified from a cluster of closely spaced frequencies. Based on the available observations, simplified models with guy lines (SPE) seems to be able to provide relatively accurate results with less computational efforts.

It is necessary to point out that the boundary conditions of the conductors in the detailed pole-line models assumed fixed ends. Realistic models should also consider soil springs. Optimizing the boundary conditions to include both the influence of neighboring structures and realistic soil conditions may also increase the accuracy of the numerical results.

It is also important to point out that because of the centrifuge effect during spun production and the prestress effects, the actual concrete modulus of elasticity in the concrete pole model may be different from the design value.

\subsection{Implications to strong ground motion impact on transmission poles}

There are two complications essentially associated with strong ground motion effects on power transmission systems: 1) soil-structure interactions associated with the support structure and 2) the transfer of energy to the conductor cables. The effect of soil-structure interactions to the transmission structure is another complication that can be highly nonlinear according to soil types. Since the conductor's resonant vibrations may be in the same range as the pole structures, El-Attar's observation [4] of strong displacements and internal forces may be due to resonance.

The simplified FE models proposed in this paper mainly concern with the structural integrity of the pole structure itself. To establish a limiting ground vibration level for these pole structures, the following process can be applied to 
the simplified FE models: 1) monitor ground vibration close to transmission structure foundations; 2) design ground motions with anticipated vibration scale; 3 ) apply the designed ground vibration as foundation excitations into the FE models to obtain structural responses; and 4) compare structural responses with transmission structure design requirements to determine safety limits due to ground movements.

For detailed ground motion design, soil-structure interactions, stress wave propagations, and dynamic load characteristics including load duration and magnitudes, should be considered. Cable-pole interactions increase complexities of structural dynamic responses and are possible reasons to introduce damages to structural components such as connectors.

\section{Conclusions}

In this paper, modal behaviors of the transmission structure were studies both experimentally and numerically. According to the research results, the following conclusions can be drawn:

1. Transmission structures have a strong coupling issue between the main structure and the cables. FE models only consider pole vibration yield natural frequencies that differ from the physical testing results; i.e. 2 nd bending mode frequency of CPA is $11.31 \%$ difference from the modal testing result (Tabl 1). Hence, cable effects cannot be neglected.

2. Detailed FE models of the coupled pole-line system yield insights into the coupling phenomena. Both the "cable-leading modes" and the "pole-leading modes" are observed in the analysis. This coupling issue makes it difficult for mode identification. The numerical results from detailed models are closer to the experimental results, although there are still some modeling uncertainties remaining.

3. Simplified models proposed by Li et al. [12] are good candidates for modeling the coupled pole-line system. The lower mode natural frequencies calculated from the simplified models of the studied pole-line system are close to experimental data and the detail modeling results.

\section{Acknowledgements}

The author would like to acknowledge the financial support of Southern Company: Southern Company Transmission System Design Committee, Mr. Charles Munden and Mr. Dennis Mize. Special appreciation is extended to Mr. Colby Galloway, Mr. Luke Stafford, Mr. Eddie Sheffield of Southern Company, Mr. Bill Kitchens of the Alabama Department of Surface Mines, and Mr. Mac Sauls and Mr. Randall Franklin of the SAULS Seismograph group. The views, opinions, findings and conclusions reflected in this publication are the responsibility of the authors only and do not represent the official policy or position of the Southern Company.

\section{References}

[1] D.E. Siskind, M.S. Stagg, J.W. Kopp and C.H. Dowding, Structure response and damage produced by ground vibraion from surface mine blasting, Report of investigations 8507, Washington D.C.: United States Bureau of Mines, 1980.

[2] Y. Momomura, H. Marukawa, T. Okamura, E. Hongo and T. Ohkuma, Full-scale measurements of wind-induced vibration of a transmission line system in a mountainous area, Journal of Wind Engineering and Industrial Aerodynamics 72 (1997), 241-252.

[3] A.M. Horr, A. Yibulayin and P. Disney. Nonlinear spectral dynamic analysis of guyed towers. Part II: Manitoba towers case study, Canada Journal of Civil Engineering 31 (2004), 1061-1076.

[4] M.M. El-Attar, Nonlinear dynamics and seismic response of power transmission lines, Ph.D. dissertation. McMaster University, Hamilton, Ontario, Canada, 1997.

[5] A. Ghobarah, T.S. Aziz and M. El-Attar, Response of transmission lines to multiple support excitation, Engineering Structures 18(12) (1996), 936-946.

[6] A.M. Loredo-Souza, The behavior of transmission lines under high winds, Ph.D. dissertation, The University of Western Ontario, London, Ontario, Canada, 1996.

[7] G. McClure and M. Lapointe, Modeling the structural dynamic response of overhead transmission lines, Computers and Structures $\mathbf{8 1}$ (2003), 825-834. 
[8] S.H. Venkatasubramanian, Determination of the initiating mode for transmission line galloping, Ph.D. dissertation, Kansas State University, Manhattan, Kansas, 1992.

[9] R. Karoumi, Some modeling aspects in the nonlinear finite element analysis of cable supported bridges, Computers and Structures $\mathbf{7 1}$ (1999), 397-412.

[10] S. Ozono and J. Maeda, In-plane dynamic interaction between a tower and conductors at lower frequencies, Engineering Structures $\mathbf{1 4}$ (1992), 210-216.

[11] S. Ozono, J. Maeda and M. Makino, Characteristics of in-plane free vibration of transmission line systems, Engineering Structures 10(4) (1988), 272-280.

[12] H.N. Li, W.L. Shi, G.X. Wang and L.G. Jia, Simplified models and experimental verification for coupled transmission tower-line system to seismic excitations, Journal of Sound and Vibration 286 (2005), 569-585.

[13] T.B. Lantrip, Identification of structural characteristics of spun prestressed concrete poles using modal testing methods, M.S. Thesis. Birmingham, Alabama: University of Alabama at Birmingham, 1995.

[14] S.E. Chen, C.K. Ong and K. Antonsson, Modal behaviors of spun-cast pre-stressed concrete pole structures, In: Proceeding of IMAC XXIV. Saint Louis, Missouri, USA, 2006.

[15] D. Polyzois, I.G. Raftoyiannis and S. Ibrahim, Finite elements method for the dynamic analysis of tapered composite poles, Composite Structures 43(1) (1998), 25-34.

[16] K.S. Dai and S.E. Chen, Vibration of spun-cast prestressed concrete poles, In: Proceedings IMAC - XXV, Orlando, Florida, USA, 2008.

[17] C.K. Ong, S.E. Chen, C. Galloway, C. Munden and M. Dennis, Innovative application of geophysical techniques for design of directembedded pole structures, in: Electrical Transmission Line and Substation Structures: Structural Reliability in a Changing World, R.E. Nickerson, ed., American Society of Civil Engineers, 2006, pp. 207-214.

[18] BSSC, NEHRP Recommended Provision for Seismic Regulations for New Buildings and Other Structures, (2003 ed.), Washington, DC: Building Seismic Safety Council, 2004, pp. 47-48.

[19] ANSYS, Release 10.0 documentation for ANSYS ${ }^{\circledR}$, Canonsburg, PA. SAS IP, Inc.; 2005. 

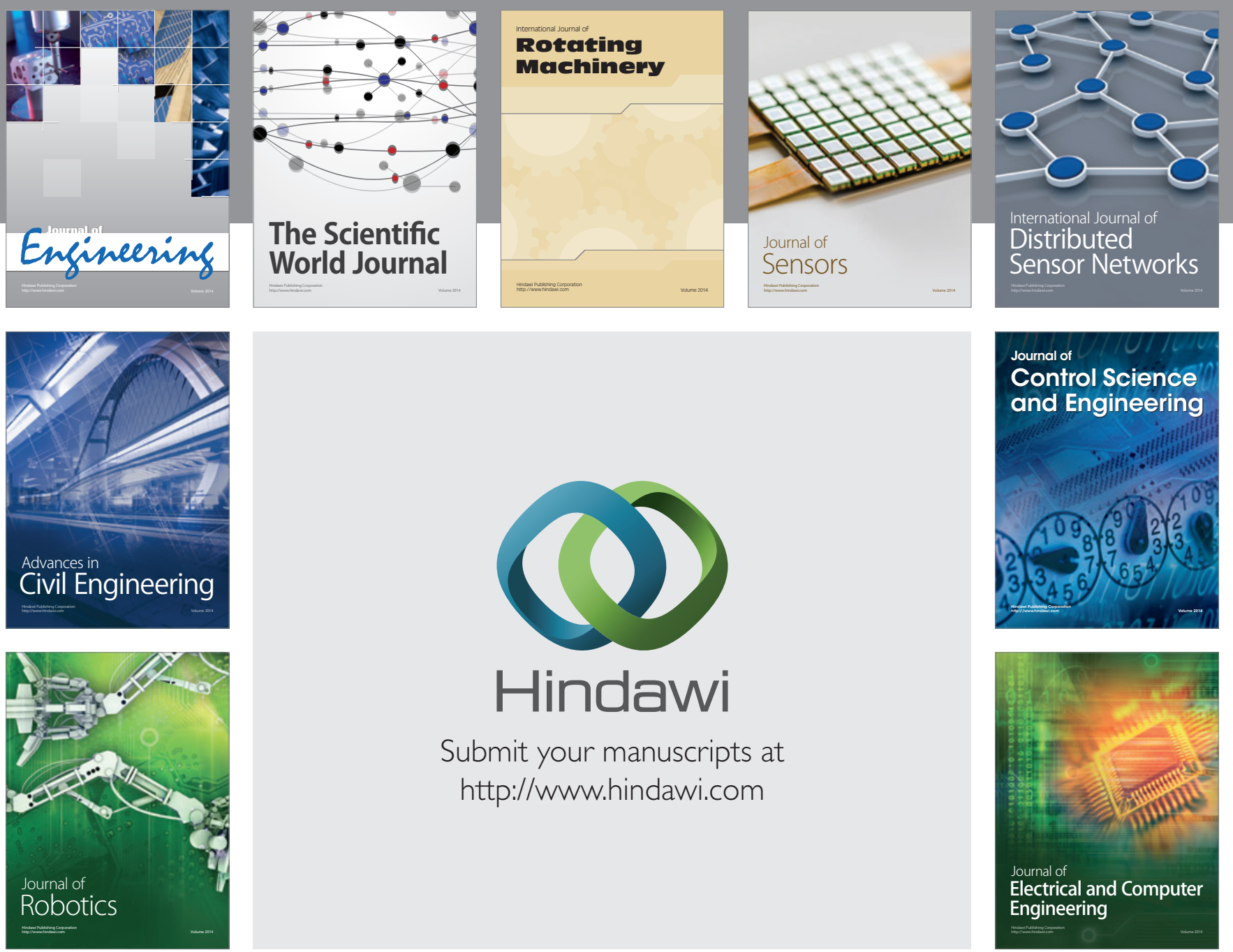

Submit your manuscripts at

http://www.hindawi.com
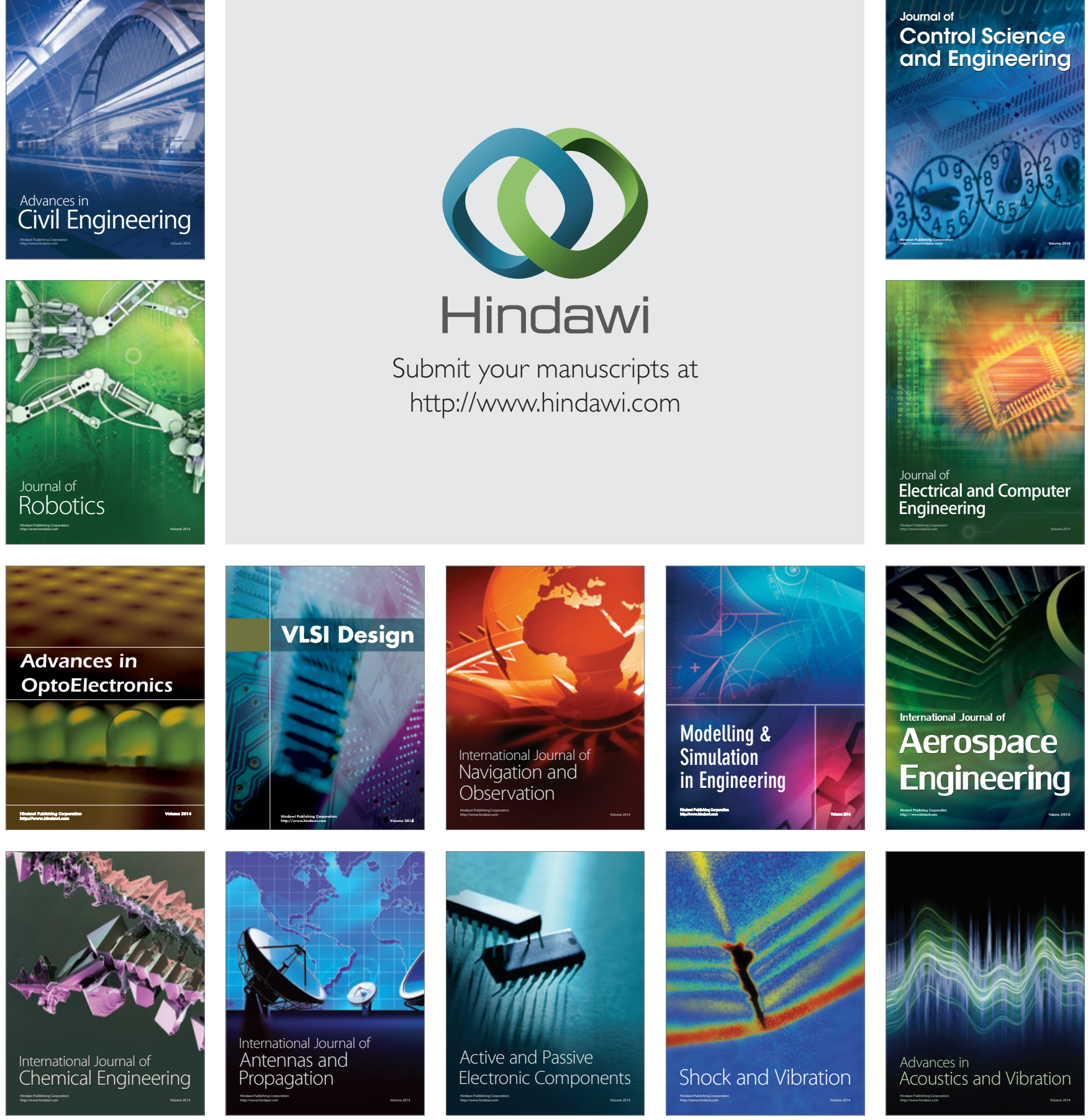